\title{
Sistema MIMO óptico inalámbrico con modulación BPSK y recepción óptima homodina tolerante a difusión de fase óptica y turbulencia atmosférica
}

\author{
MIMO Optical Wireless System with BPSK Modulation and Optimum \\ Homodyne Reception Tolerant to Optical Phase Diffusion and \\ Atmospheric Turbulence
}

\author{
Arvizu-Mondragón Arturo \\ Centro de Investigación Científica y de Educación Superior de \\ Ensenada \\ Correo:arvizu@cicese.mx \\ Santos-Aguilar Joel \\ Centro de Investigación Científica y de Educación Superior de \\ Ensenada \\ Correo:jsantos@cicese.mx
}

\author{
Cabrera-Nava Alejandro \\ Huawei Technologies de Mexico \\ Network Technical Services Department, Optical Implementation \\ Correo: alex.cabrera.nava@gmail.com
}

Información del artículo: recibido: julio de 2015, aceptado: septiembre de 2015

\section{Resumen}

En este trabajo se presenta un sistema de comunicaciones MIMO óptico inalámbrico con modulación binaria de la fase óptica, el cual emplea diversidad espacial con recepción óptica homodina, estimación óptima, postdetección de la fase óptica y combinador lineal de máxima verosimilitud. El uso de una configuración de diversidad espacial permite obtener una mayor tolerancia a los efectos que la turbulencia óptica provoca sobre la señal de información, mientras que la estimación de fase óptima compensa la degradación sobre la tasa de bit erróneo ocasionada por el ruido de fase, inherente a las fuentes ópticas comúnmente utilizadas en las telecomunicaciones. Este enfoque, no se ha abordado previamente desde el punto de vista experimental, por ello consideramos que esta es la contribución más importante de este trabajo. Se presenta la implementación y caracterización de nuestro sistema en un montaje experimental simplificado.

\section{Descriptores:}

- MIMO óptico

- comunicaciones ópticas inalámbricas

- recepción homodina

- turbulencia óptica

- variables de estado 


\begin{abstract}
This paper presents an optical wireless MIMO communication system with binary modulation of the optical phase, which uses spatial diversity, optical homodyne reception, a post-detection stage for the optimal estimation of the optical phase, and maximum-likelihood linear combining stage, The use of a spatial diversity configuration enables to get a higher tolerance to the effects that the turbulence causes on the optical information signal while the optimal phase estimation stage compensates the degradation over the bit error rate caused by the phase-noise inherent to the optical sources commonly used in telecommunications. This approach, to the best of our knowledge, has not previously been addressed from the experimental point of view; we think that this is the more important contribution of our work. So, we present the implementation and characterization of our system in a simplified experimental set-up.
\end{abstract}

\section{Introducción}

La importancia de los sistemas de comunicaciones ópticas inalámbricas (OWC, Optical Wireless Communications o FSO, Free Space Optics) radica en ser un complemento a los sistemas de radiofrecuencia (RF) y redes de fibra óptica, con ventajas tales como altas tasas de transmisión (inherentes a portadoras ópticas), no requerimiento de licencia para operar e inmunidad a interferencia electromagnética. Estos sistemas son atractivos también en aplicaciones militares o comerciales tales como la "última (o primera) milla", enlaces para casos de emergencia, transmisión de información con alta seguridad, comunicación óptica espacial, entre otros.

Los sistemas modernos de comunicación óptica en espacio libre pueden remontarse al fotófono inventado por Alexander Graham Bell en 1881 (Goyal et al., 2015; Bell, 1881) que no fue comercializado por inexistencia en dicha época de fuentes ópticas coherentes y requerir operar en línea de vista, entre otras complicaciones. Para los años 60 del siglo pasado, el interés por esta tecnología resurgió por la aparición de fuentes ópticas coherentes (láseres) y empezó a desarrollarse paralelamente a los sistemas por fibra óptica (Lighpointe, 2015). Sin embargo, la señal óptica propagándose en un medio atmosférico no guiado (altamente no predecible), se puede afectar por nubes, niebla y gases que dispersan el haz láser mientras que la turbulencia atmosférica perturba su intensidad y fase, pudiendo provocar desvanecimientos para turbulencia fuerte (Popoola et al., 2008). Los efectos de la turbulencia óptica pueden mitigarse mediante diferentes estrategias:

a) incremento de la potencia óptica transmitida

b) diversidad temporal, frecuencial o espacial (Farid et al., 2012) c) procesamiento digital de señales postdetección óptica coherente

d) técnicas de estimación de las características del canal (Goyal et al., 2015)

e) usar sistemas híbridos FSO/RF (Lighpointe, 2015)

f) técnicas de codificación/corrección de errores (Goyal et al., 2015)

Aquí se aborda teórica-experimentalmente la diversidad espacial en conjunto con técnicas óptimas (de máxima verosimilitud) de combinación lineal de señal postdetección y estimación óptima de fase óptica, lo cual no se ha reportado previamente, aunque sí se han reportado resultados de simulación (Arvizu et al., 2010). Así, se considera que una de las principales aportaciones del presente trabajo es el desarrollo y caracterización experimental del subsistema mencionado, mostrando un desempeño satisfactorio.

Para los sistemas FSO que emplean diversidad espacial existen diversos métodos de combinación lineal de señal postdetección óptica:

a) esquema SC (Selection Combining): la señal recibida con el mayor nivel de potencia se selecciona y procesa para recuperar los datos

b) esquema MRC (Maximal Ratio Combining): consiste en procesar la señal resultante de sumar todas la señales recibidas. Estos métodos de combinación son idóneos para información transmitida en intensidad del campo óptico, sin embargo, cuando la información está en la fase, frecuencia o estado de polarización de la luz y el observable eléctrico es resultado de un "batimiento" óptico coherente es mejor utilizar otras técnicas de combinación tales como el combinador lineal óptimo (de acuerdo con el criterio de máxima verosimilitud) post-detección 
(PDLC), propuesto originalmente por Van Trees en 1971 para canales de radio con desvanecimiento.

Si bien la investigación y desarrollo de sistemas FSO es relevante desde los años 60 del siglo pasado, y se han abordado aspectos como el modelado del canal, esquemas de modulación-demodulación, esquemas de detección, optimización de longitudes de onda, repetidores (relay-assisted, Kashani et al., 2013), entre otros, a la fecha estos sistemas son un área activa de investigación (por ejemplo, Goyal et al., 2015; Khalighi y Uysal, 2014; Prabu y Kumar, 2015). En particular, dentro de este ámbito, en el presente trabajo se aborda el desarrollo experimental de un sistema óptico MIMO, técnica ampliamente conocida en el dominio de RF y aunque ya se han estudiado diversos aspectos en el ámbito de FSO, aquí nos enfocamos en una solución "óptima" de acuerdo con la teoría estadística de las comunicaciones, donde el criterio de optimización se basa en un modelo apegado a las características del canal óptico atmosférico empleando una descripción en variables de estado (Snyder,1969). En contraste, en los sistemas de RF se usa normalmente el modelo "señal más ruido" donde el único ruido a considerar es del tipo aditivo, mientras que en sistemas ópticos BPSK además del ruido aditivo (de fotodetección) existe el ruido de fase que es parte del argumento de la portadora y por lo tanto, es no lineal.

Posibles aplicaciones de sistemas MIMO BPSK ópticos son enlaces ópticos de alta velocidad operando en ambientes de turbulencia baja cercana a alta turbulencia, por ejemplo, entre una estación óptica terrena y un satélite o enlaces ópticos horizontales en espacio libre de mediana distancia y alta velocidad. Se muestra que con la configuración MIMO con detección coherente, y combinador PDLC implementada, se obtiene mayor tolerancia a turbulencia óptica en comparación con un sistema de un solo haz de transmisión y una apertura receptora. Es pertinente mencionar que Niu et al. (2011) reporta el uso de un esquema similar, sin embargo, este esquema difiere en el hecho de que se busca optimizar tanto la etapa de recuperación de los datos como ponderando cada una de las ramas del sistema MIMO de acuerdo con la probabilidad de ocurrencia (Arvizu et al., 2010).

\section{Diversidad espacial en sistemas ópticos con detección homodina}

Se conoce ampliamente que la diversidad espacial permite mitigar los efectos atmosféricos al transmitir $M$ versiones de la señal deseada usando $M$ diferentes canales, buscando que cada canal sufra desvanecimientos diferentes.
Las técnicas de diversidad empleadas en sistemas inalámbricos son:

a) diversidad temporal: la información se envía empleando la misma frecuencia de portadora, pero en diferentes periodos de tiempo; cada símbolo se transmite $M$ veces

b) diversidad frecuencial: la transmisión de información se realiza a través de $\mathrm{M}$ diferentes portadoras, así, se realiza una transmisión simultánea de los símbolos

c) diversidad espacial: consiste en usar múltiples "copias" de la señal transmitida. Usando múltiples antenas en las estaciones transmisoras o receptoras, con espaciamiento entre ellas tal que las señales recibidas por cada una tengan diferentes desvanecimientos.

En este trabajo se presenta un sistema de comunicaciones con modulación binaria de fase óptica y detección homodina. Para este tipo de sistemas se pueden emplear receptores coherentes con un área colectora única o sistemas con un área efectiva de colección múltiple empleando arreglos de varios receptores. En los sistemas de RF suelen llamarse sistemas MIMO (Multiple Input-Multiple Output), término adoptado también en sistemas FSO aunque también se emplea el término MBT-MAR (Multiple Beam Transmitter-Multiple Aperture Receiver). Una de las ventajas asociadas al utilizar diversidad espacial es que el número de "partículas de turbulencia" sobre cada una de las trayectorias o subaperturas es menor respecto a las de un esquema de apertura única. Las señales provenientes de cada receptor se pueden combinar electrónicamente para mejorar el proceso de detección. De manera general, el desempeño de los sistemas de combinación mejora en función del incremento del número de subaperturas (Belmonte y Kahn, 2009). Es importante remarcar que la conveniencia del uso de un esquema de diversidad espacial consiste en recibir redundantemente la misma señal de información en los canales del sistema y beneficiarse de la baja probabilidad de grandes desvanecimientos simultáneos en cada uno de los diversos canales.

\section{Esquema experimental}

En este trabajo se emplea una configuración de recepción MIMO que incluye etapas de estimación-correlación (para recuperación de los datos) para cada antena receptora del arreglo con diversidad espacial, donde se procesarán tantos observables eléctricos $\left(\mathrm{V}_{\mathrm{O}}(\mathrm{t})\right)$, como antenas receptoras se tengan. Se propone un algoritmo 
que asigna un peso, $\mathrm{P}_{\mathrm{k}}$ (ecuación 2) a cada rama, este peso corresponde al cociente entre la intensidad recibida en cada rama óptica $I_{k}$ y la intensidad total recibida $\mathrm{I}_{\text {Total }}$ (ecuación 1) por todas las ramas. El asignar este peso a cada rama es para ponderar cada una de las señales arribando a la estructura receptora, así, la que tenga el mayor peso será aquella con menor probabilidad de desvanecimiento (pues esta es la que tiene mayor intensidad relativa).

$$
\begin{aligned}
& I_{\text {Total }}=I_{1}+I_{2}+\ldots I_{k} \\
& P_{k}=\overline{I_{k}} / \overline{I_{\text {Total }}}
\end{aligned}
$$

Tras multiplicar el valor del estimado de fase de máxima verosimilitud (ML) por el peso específico de cada rama, cada bloque estimador tendrá un par de salidas, el estimado ponderado para " 1 ", $\mathrm{PML}_{1}$, y para "0", $\mathrm{PML}_{0}$. En la etapa siguiente se asigna a $\mathrm{S}_{0}$ el resultante de la suma de todos los estimados ponderados de " 0 " y a $S_{1}$ la correspondiente suma de " 1 ".

$$
\begin{aligned}
& \mathrm{S}_{0}=\mathrm{PML}_{0,1}+\mathrm{PML}_{0,2}+\ldots \mathrm{PML}_{0, k} \\
& \mathrm{~S}_{1}=\mathrm{PML}_{1,1}+\mathrm{PML}_{1,2}+\ldots \mathrm{PML}_{1, k}
\end{aligned}
$$

Finalmente se comparan $S_{0}$ y $S_{1}$, si $S_{0}$ resulta mayor entonces el dato estimado $D_{k}=0$, en caso contrario, $\left(S_{1}\right.$ es mayor) $D_{k}=1$ (figura 1a).

\section{Estimador-correlacionador}

A continuación se describe su operación por conveniencia, para un solo canal. En el receptor se tienen las siguientes señales

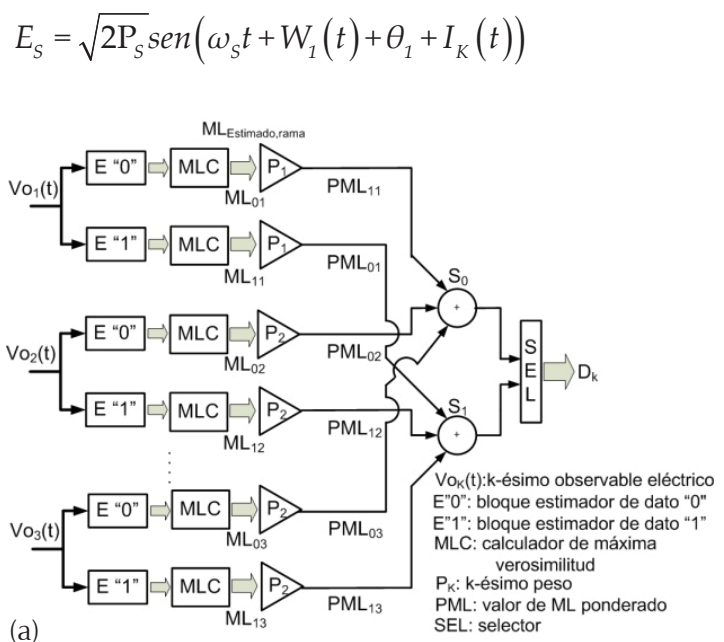

$$
V_{O}=S\left(x, t, I_{K}\right)+n(t)
$$

Figura 1. a) Diagrama a bloques del estimador de fase con diversidad espacial grado $k$, b) esquema receptor con estimadorcorrelacionador 
$S\left(x, t, I_{K}\right)=\sqrt{2 P_{H}} \operatorname{sen}\left(\omega_{F I} t+x+I_{K}\right)$

$\sqrt{2 \mathrm{P}_{H}}=\sqrt{(4 A r \Re)^{2} P_{S} P_{O L}}=\sqrt{2\left(8 \mathrm{~A}^{2} r^{2} \mathfrak{R}^{2} P_{S} P_{O L}\right)}$

redifiniendo el observable eléctrico

$V_{O}(t)=r(t)=\frac{d y(t)}{d t}$

$d y(t)=S\left(x, t, I_{K}\right) d t+\sqrt{\frac{N_{O}}{2}} d u(t)$

$n(t)=\sqrt{\frac{N_{O}}{2}} d u(t)$

Usando las ecuaciones (9)-(12) se pueden obtener las ecuaciones de estimación de fase $x^{*}(t)$ y varianza $v^{*}(t)$ requeridas por el estimador-correlacionador (Snyder, 1969), donde fLT es el ruido de fase total de la señal óptica de batimiento. Con estas expresiones se realiza la estimación del dato transmitido. La entrada de los bloques "estimador" (figura 1b) es el observable eléctrico (ecuación 7).

$$
\begin{aligned}
& x(t)^{*}=\int\left[\frac{\sqrt{8 \mathrm{P}_{H}}}{N_{O}} v^{*} \exp \left(\frac{-v^{*}}{2}\right) \cos \left(x+I_{K}\right) r(t)\right] d t- \\
& \int\left[\frac{2 \mathrm{P}_{H}}{N_{O}} v^{*} \exp \left(-v^{*}\right) \operatorname{sen}\left(2\left(x+I_{K}\right)\right)\right] d t \\
& v^{*}(t)=-\int\left[\frac{\sqrt{8 \mathrm{P}_{H}}}{N_{O}}\left(v^{*}\right)^{2} \exp \left(\frac{-v^{*}}{2}\right) \operatorname{sen}\left(x+I_{K}\right) r(t)\right] d t- \\
& \int\left[\frac{4 \mathrm{P}_{H}}{N_{O}}\left(v^{*}\right)^{2} \exp \left(-v^{*}\right) \cos \left(2\left(x+I_{K}\right)\right)\right] d t+\int \frac{1}{\left(2 \pi f_{L T}\right)^{-1}} d t
\end{aligned}
$$

Se necesitan dos bloques, pues existen dos posibilidades en los datos transmitidos para " 1 ” o “ 0 ” ( $\mathrm{I}_{\mathrm{K}}=0$ o $\left.\pi\right)$ respectivamente, cuya salida es

$$
S^{*}\left(x, t, I_{K}\right)=\exp \left(\frac{-v^{*}(t)}{2}\right) S\left(x^{*}, t, I_{K}\right)
$$

A continuación, los calculadores de máxima verosimilitud (ML) realizan una correlación del estimado con el observable, obteniendo el máximo en la probabilidad condicional inducida en el observable, dado el estimado calculado por cada estimador. La correlación debe realizarse para cada dato, por tanto está definida en un intervalo de tiempo equivalente al periodo de bit $(\mathrm{T})$ de la secuencia de datos transmitidos.

$$
\lambda\left(I_{K}\right)=\int_{0}^{T} S\left(t, x, I_{K}\right) V_{O}(t) d t
$$

Las salidas $\lambda\left(I_{K}\right)$ de los calculadores ML se comparan en un "selector" que elige la mayor de las magnitudes proporcionadas por los calculadores ML, tanto para el estimado " 0 " como para el " 1 ". La salida del "selector" es el dato estimado $\left(I_{K}^{*}\right)$.

\section{Cámara para generación de turbulencia}

En el canal atmosférico se presentan dos fenómenos, en general, esparcimiento y centelleo inducido por turbulencia atmosférica (Hajjarian y Fadlullah., 2009). Las inhomogeneidades en temperatura y variaciones de presión en la atmósfera provocan variaciones en el índice de refracción, modificando las señales ópticas propagándose a través de dicho medio; este efecto se conoce como turbulencia óptica atmosférica.

Las fluctuaciones en la señal detectada provocadas por las variaciones aleatorias en el índice de refracción a lo largo del canal se conocen como centelleo. Los efectos de las fluctuaciones causadas por la turbulencia atmosférica pueden estimarse mediante la relación con el parámetro de la constante de la función de estructura del índice de refracción del aire, $C_{n}^{2}$, relacionado a su vez con la temperatura y presión atmosférica como

$$
C_{n}^{2}=\left(79 \times 10^{-6} \frac{P}{T}\right)^{2} C_{T}^{2}
$$

donde

$P[$ milibars $]=$ presión atmosférica

$T\left[{ }^{\circ}\right.$ Kelvin] = temperatura

$C_{T}^{2}\left[\mathrm{~m}^{-2 / 3}\right]=$ constante de la función de estructura

Otro parámetro relacionado con la intensidad de la turbulencia es la varianza de Rytov que involucra la longitud de la trayectoria recorrida por el haz y longitud de onda $(\lambda)$ en cuestión

$\sigma_{R}^{2}=1.23 C_{n}^{2} k^{76} L^{11 / 6}$ 
donde $\mathrm{k}=2 \pi / \lambda$ número de onda óptico y $\mathrm{L}$ [metros] distancia entre estación emisora y receptora. El índice de centelleo es otro parámetro asociado a la turbulencia atmosférica y al centelleo

$$
\sigma_{I}^{2}=\frac{\left\langle I^{2}\right\rangle-\langle I\rangle^{2}}{\langle I\rangle^{2}}=\frac{\left\langle I^{2}\right\rangle}{\langle I\rangle^{2}}-1
$$

Aquí I = intensidad de la señal óptica y el operador $<>$ denota el valor promedio en el intervalo de tiempo de monitoreo. Para turbulencia débil (Andrew y Phillips, 2005)

$\sigma_{I}^{2}=\sigma_{R}^{2}=1.23 C_{n}^{2} k^{76} L^{11 / 6}$

para turbulencia media y fuerte

$\sigma_{I}^{2}=0.4 \sigma_{R}^{2}$

Para caracterizar el esquema experimental en condiciones de turbulencia controlada dentro del laboratorio, se usó una cámara de turbulencia (figura 3a) consistente de una caja de material térmico con cuatro agujeros, conteniendo en su interior un elemento calefactor y dos ventiladores con velocidades ajustables. A través de los agujeros se hace pasar un haz luminoso, el cual puede duplicar su trayectoria empleando un par de espejos y al final de la trayectoria óptica se utiliza un fotodiodo para evaluar el nivel de turbulencia dentro de la cámara. Es conveniente calibrar la cámara basándose en las ecuaciones (19) y (22) de donde se obtiene la figura $3 \mathrm{~b}$ para un enlace (por conveniencia) de $\mathrm{L}=353 \mathrm{~m}$ y longitud de onda $\lambda=550 \mathrm{~nm}$ (verde), el cual se implementa y evalúa prácticamente (figuras $4 \mathrm{a}$ y b) con los resultados mostrados en la figura 5a:

a) señales adquiridas por un osciloscopio digital

b) histograma generado con los datos adquiridos c) funciones de densidad de probabilidad lognormal generadas con los parámetros de varianza obtenidos.

En la tabla 1 se resumen los resultados obtenidos experimentalmente, así como los valores teóricos respectivos. Para evaluar los niveles de turbulencia obtenibles con la cámara de turbulencia se utilizó la misma fuente óptica y detectores del experimento en espacio libre y el valor máximo de centelleo fue 0.317 (figura $5 b$ ), utilizando este valor y la expresión (22) se determinó que la cámara de turbulencia es útil para emular turbulencia de diversos regímenes (débil, media y fuerte). Pero la intensidad de una señal óptica no es el único parámetro que se ve afectado por la turbulencia atmosférica, el estado de polarización (SOP) también puede sufrir afectaciones. Existen cuatro posibles impactos de un medio aleatorio como la atmósfera sobre la polarización de un haz láser: la despolarización parcial o total de la luz, que la atmósfera actúe como un medio birrefringente causando que las componentes $x$ y y del campo eléctrico viajen a velocidades distintas, la rotación en el ángulo de la polarización lineal y por último que la atmósfera se comporte como un filtro permitiendo así la transmisión de un SOP en particular y atenuando el respectivo SOP ortogonal. La cámara de turbulencia se caracterizó también en función de dos parámetros asociados al estado de polarización de una señal; la variable $\eta$, (figura 6a), que simboliza el ángulo de polarización lineal y el parámetro DOP que corresponde al grado de polarización. En las figuras 6 b y c se muestran los gráficos desplegados por el analizador de polarización de espacio libre (RPA2000, Instrument Systems) empleado en la caracterización. Para cada figura: sección a) muestra el comportamiento del campo eléctrico, es decir, el tipo de polarización que la señal presenta, la sección b) muestra los cambios del ángulo de la elipse de polarización en el tiempo (ángulo azimut (ๆ)), del grado de polarización (DEP) y de las variaciones de intensidad, y la sección c) despliega los datos numéricos promedio.
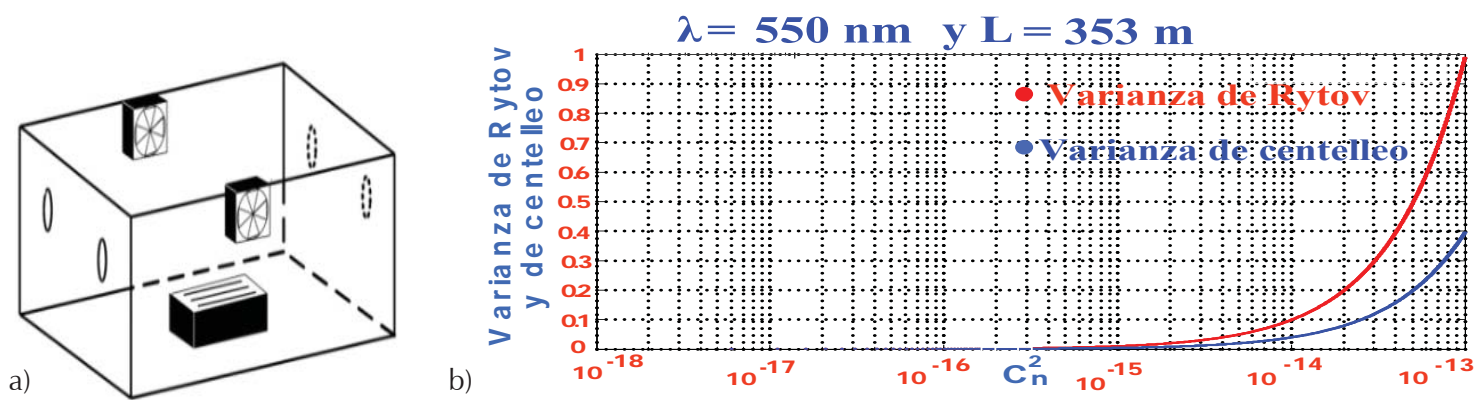

Figura 3. a) Cámara de turbulencia, b) varianzas de Rytov y centelleo para $L=353 \mathrm{~m}$ y $\lambda=550 \mathrm{~nm}$ 
Tabla 1. Comparación entre los índices de centelleo de simulación y prácticos para $\mathrm{L}=353 \mathrm{~m}$

\begin{tabular}{ll}
\hline \multicolumn{1}{c}{$\lambda$} & \multicolumn{1}{c}{$550 \mathrm{~nm}$ (verde) } \\
\hline$\sigma_{I}^{2}$ teórico & $0<\sigma_{I}^{2}<0.125$ \\
$\sigma_{I}^{2}$ experimental & 0.09 \\
\hline
\end{tabular}

Con la cámara de turbulencia apagada la señal presenta un estado de polarización elíptico (sección a), la variación del ángulo azimut es $\Delta \eta=1^{\circ}$, el grado de polarización oscila entre 85 y 95\% y el nivel de intensidad varía alrededor de $1 \mathrm{~dB}$ (sección $\mathrm{b}$ ). Con la presencia de turbulencia atmosférica inducida por la cámara, el estado de polarización permanece elíptico (sección a), los cambios relevantes se presentan en la sección b), donde es posible apreciar que el valor del ángulo azimut $\eta$ presenta oscilaciones entre los $73^{\circ}$ y $80^{\circ}$, es decir $\Delta \eta=7^{\circ}$, mientras que el grado de polarización, DEP, conserva el mismo comportamiento que el observado en ausencia de turbulencia atmosférica inducida por la cámara. Se nota que por efecto de la turbulencia inducida no se presentaron cambios en el estado de polarización; el es- tado de polarización elíptico se conservó y la elipticidad prácticamente no sufrió cambios, conservando un valor semejante $\left(17^{\circ}\right)$. Si bien las gráficas muestran variaciones en el grado de polarización no es válido asociar esta oscilación a la turbulencia inducida, pues dichas variaciones están también presentes en las pruebas realizadas sin presencia de turbulencia. El efecto que es notablemente provocado por la turbulencia atmosférica inducida por la caja es la variación del ángulo azimut $\eta$; este rango de variación es similar al reportado por otros estudios (Grosinger, 2008).

\section{Arreglo experimental planteado}

Basándonos en el sistema de la figura $1 \mathrm{~b}$ se implementó un esquema experimental como el que se muestra en la figura 7 , donde se tiene la transmisión inalámbrica de una señal óptica cuya fase es modulada (BPSK) por una señal de datos. La señal modulada pasó a través del dispositivo emulador de turbulencia atmosférica y posteriormente se capturó en la etapa de recepción. Por
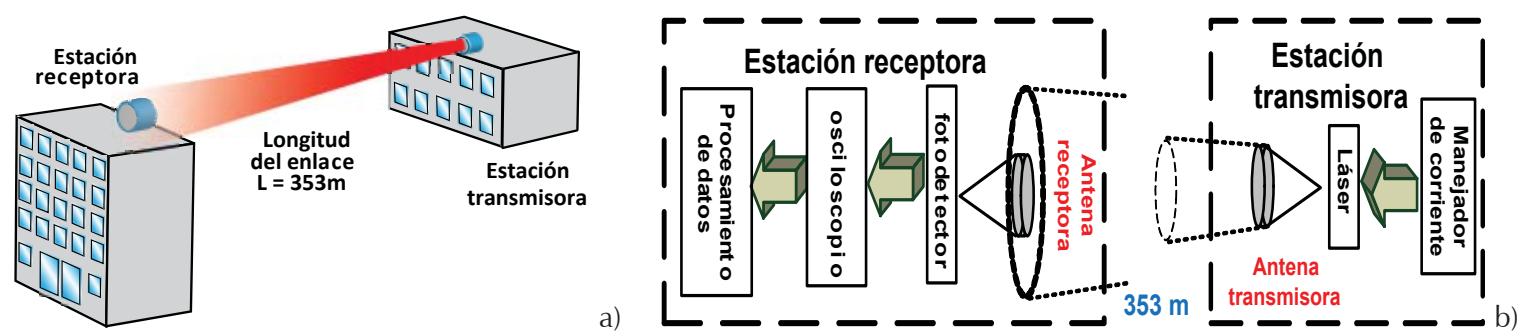

Figura 4. Enlace óptico en espacio libre para la medición experimental del índice de centelleo, a) diagrama general, b) detalle de estaciones transmisora y receptora
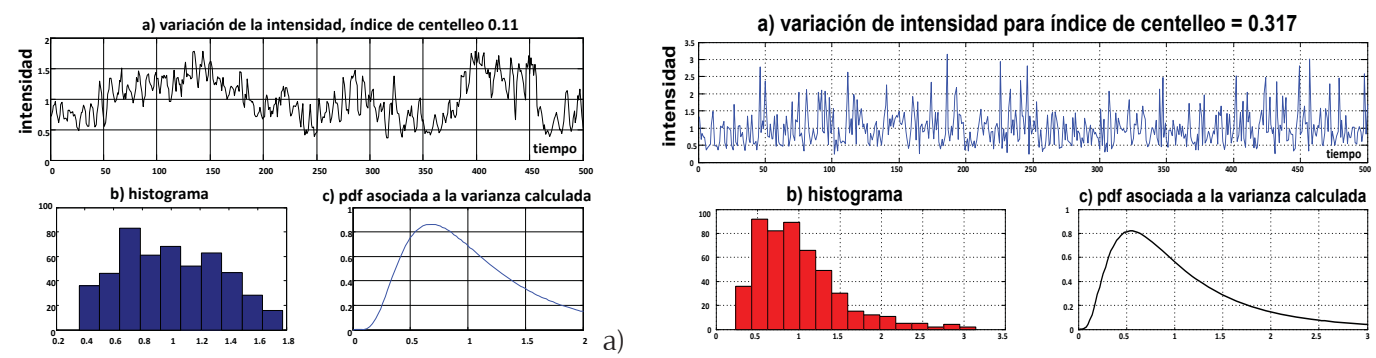

Figura 5. a) Variación de intensidad en un enlace con $\lambda=550 \mathrm{~nm}$, b) centelleo generado con cámara de turbulencia
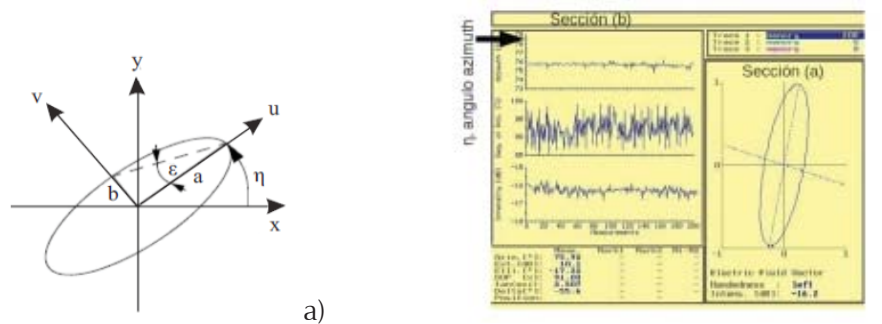

b)

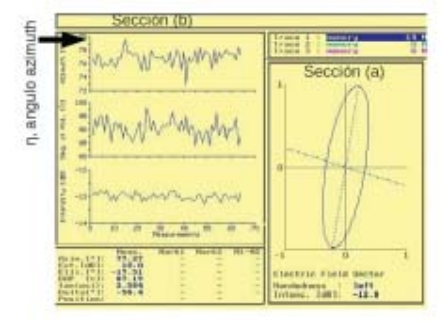

c)

Figura 6. a) Parámetro a evaluar en el estado de polarización. Medición de SOP con cámara de turbulencia: b) apagada, c) encendida 
conveniencia, el esquema empleado es el de un sistema coherente autohomodino, la configuración del receptor es balanceada y para la adquisición de los datos se utilizó un osciloscopio digital. Dichos datos se procesan fuera de línea para la determinación final de la señal recibida. La señal portadora se genera por un dispositivo láser, esta señal se dividide por un acoplador de 90/10, pues como se mencionó anteriormente, el esquema es autohomodino así que parte de la potencia es modulada $\left(E_{S}\right)$ y la parte restante se emplea como oscilador local $\left(\mathrm{E}_{\mathrm{OL}}\right)$. Los datos se generan de manera pseudoaleatoria, el dispositivo modulador (de espacio libre) es el encargado de modular la fase de la señal portadora en función de la secuencia de datos previamente mencionada, la señal eléctrica de datos se acondiciona usando un "driver" para que el modulador opere correctamente. El controlador de polarización colocado en la entrada óptica del modulador se utiliza para obtener el mejor desempeño del modulador de fase. La salida óptica del modulador de fase proporciona la señal de información $\mathrm{E}_{\mathrm{S}}$ que viajará por el canal atmosférico (emulado en este caso por la cámara de turbulencia). Esta señal $\left(E_{S}\right)$ se captura por una lente tipo GRIN fibrada y es una de las señales de entrada al acoplador encargado de la mezcla de la señal de información $\mathrm{E}_{\mathrm{S}} \mathrm{y}$ oscilador local $\mathrm{E}_{\mathrm{OL}}$, esta última se inserta en el otro puerto de entrada del acoplador de $3 \mathrm{~dB}^{\prime}$ s. Es necesaria la utilización de un controlador de polarización en cada una de las entradas del acoplador para obtener una correcta mezcla de las señales $\mathrm{E}_{\mathrm{S}}$ y $\mathrm{E}_{\mathrm{OL}}$. Una vez que se ha realizado dicha mezcla, cada una de las salidas del acoplador $\left(E_{1}\right.$ y $\left.E_{2}\right)$ se inserta en los fotodetectores, las corrientes generadas por estos son utilizadas para generar el observable eléctri$\mathrm{co}, \mathrm{Vo}(\mathrm{t})$, que es la entrada correspondiente al bloque de procesamiento digital diseñado, el estimador-correlacionador. La señal portadora se genera por una fuente laser semiconductor fibrado con ancho de línea angosto, que opera en el modo de corriente continua (CW). Esta señal se divide por un acoplador óptico 90/10, del cual se obtendrán las señales necesarias para un esquema de recepción autohomodino. La señal óptica de mayor potencia se modula en fase mediante un modulador externo para obtener $\left(\mathrm{E}_{\mathrm{S}}\right)$ y la de menor potencia será la señal que actuará como oscilador local en el esquema de recepción ( $\left.\mathrm{E}_{\mathrm{LO}}\right)$.

Los datos que se envían en el enlace se generan de forma pseudoaleatoria por un generador de datos a una velocidad de $10 \mathrm{Mbps}$, estos datos se acoplan al modulador electroóptico con un amplificador de potencia (driver), el cual tiene también la función de acoplar impedancias entre el generador de datos y el modulador de fase, que es un dispositivo electroóptico que por medio de un cristal birrefringente es capaz de modificar la fase de la señal óptica en función del voltaje de entrada. El modulador es un material birrefringente, por lo que es necesario tener una polarización lineal de entrada que coincida con el eje electroóptico con mayor índice de modulación, es por esta razón que es indispensable un controlador de polarización a su entrada. La salida óptica del modulador entrega una portadora óptica $\left(E_{S}\right)$ cuya fase es la señal de información. Esta señal se propaga por un canal atmosférico turbulento simulado en este caso por una cámara de turbulencia que se adquiere por una antena óptica tipo GRIN acoplada a fibra óptica. Un acoplador óptico de $3 \mathrm{~dB}$ se utiliza en la etapa receptora para mezclar la señal de datos con la del oscilador local. Para que la mezcla de las señales ópticas sea la adecuada es necesario que las polarizaciones de las dos señales coincidan por lo que se colocan dos controladores de polarización a la entrada del acoplador en la etapa de recepción. A la salida del acoplador se obtienen dos señales que son la mezcla de las señales ópticas con una diferencia de fase de 180 grados entre las señales en una de las ramas, es por ello que a este dis-

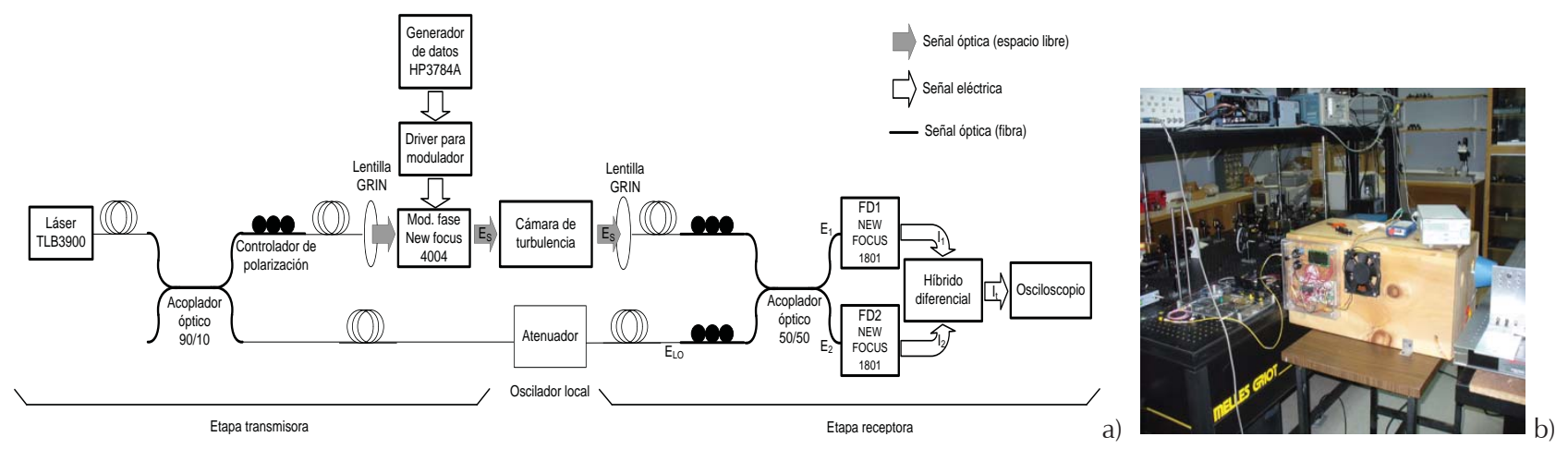

Figura 7. Esquema del sistema de comunicaciones ópticas inalámbricas implementado, a) diagrama, b) fotografía del montaje experimental 
positivo también se le llama híbrido de 180 grados. El batimiento de las señales ópticas en el acoplador produce una modulación de intensidad generando las señales $E_{1} \mathrm{y}_{2}$, las cuales se fotodetectan por bloques fotorreceptores que producen las corrientes que se utilizarán para generar los observables eléctricos $\mathrm{Vo}(\mathrm{t})$, que son la entrada correspondiente al bloque de procesamiento digital diseñado, el estimador-correlador. El arreglo presenta pérdidas de potencia óptica inherentes al sistema, en la etapa emisora las pérdidas se deben principalmente al acoplamiento entre la salida del láser fibrado y el modulador electroóptico. En la etapa receptora, en un medio sin turbulencia, las pérdidas se ocasionan por la divergencia del haz al propagarse, la antena receptora por lo regular es entonces de menor tamaño que la mancha del haz después de haberse propagado en el medio atmosférico y no es posible colectar la totalidad de la potencia emitida. En presencia de turbulencia las pérdidas pueden llegar a ser totales debido al medio atmosférico turbulento. Adicionalmente los empalmes y acoplamientos ópticos introducen pérdidas en el sistema, que si están adecuadamente realizados son despreciables. Las antenas ópticas implementadas (figura 8a) son lentes esféricos que se encuentran disponibles comercialmente. Para obtener resultados teóricos de su desempeño, se simulan mediante el software profesional de diseño óptico, ZEMAX (figura 8b).

\section{Pruebas experimentales}

Aunque en este trabajo no se llevó a cabo la implementación "completa" de un sistema de comunicaciones ópticas de diversidad espacial en "tiempo real" sí se desarrolló una estrategia para emular dicho esquema. Basado en el concepto de la diversidad espacial que considera que cada uno de los haces del transmisor MIMO sufrirá un efecto diferente de turbulencia se emula dicho efecto empleando un solo transmisor y un solo receptor, pero realizando pruebas para diferentes niveles de turbulencia con el mismo patrón de datos para todos los casos y adquiriéndolos mediante un osciloscopio digital para el procesamiento fuera de línea. De este modo se adquirieron dos señales de información para 3 niveles de turbulencia obtenidos mediante la cámara de turbulencia débil $\left(\sigma_{I}^{2}=0.087\right)$, media $\left(\sigma_{I}^{2}=0.168\right)$ y fuerte $\left(\sigma_{I}^{2}=0.341\right)$. Las figuras 9a-c corresponden a diagramas de ojo generados con capturas de datos recibidos bajo dichos niveles de turbulencia. Por otro lado, de acuerdo con Arvizu et al. (2010) para estos valores de turbulencia, con el esquema utilizado (PDLC-SV, es decir, “combinación lineal post-detección - variables de estado") se tiene para un valor de ruido de fase bajo, (aproximadamente de $10 \mathrm{KHz}$ ) que nuestro esquema presentaría una tasa de bit erróneo (BER) muy bajo (alrededor de $10^{-10}$ ) el cual corresponde al diagrama de ojo de la figura 9a. Sin embargo, ya que los láseres empleados en nuestro esquema tienen niveles de ruido mayores (alrededor de $10 \mathrm{MHZ}$ ), esto conduce a una degradación en el BER, obteniéndose los diagramas de ojo $9 \mathrm{~b}$ y $9 \mathrm{c}$ de nuestro trabajo que corresponden a valores de BER mayores.

Una vez que se contaba con señales adquiridas bajo cada uno de los regímenes de turbulencia mencionados, el paso siguiente consistió en "enfasarlos" antes de pasarlos por el estimador-correlacionador que se implementó en SIMULINK (figura 10) como etapa de procesamiento fuera de línea (no en "tiempo real"). La magnitud de los valores de las señales $\mathrm{ML}_{0}$ y $\mathrm{ML}_{1}$ se almacenan en un vector al término de cada periodo de bit; tras compararlos uno a uno, aquel que resulte mayor corresponde al dato estimado. La notación empleada para referir y distinguir a cada una las tramas adquiridas es la siguiente:

a) $\mathrm{D} \alpha, \mathrm{D} \beta$ señales con nivel bajo de turbulencia

b) $\mathrm{M} \alpha, \mathrm{M} \beta$ con turbulencia media

c) $\mathrm{F} \alpha, \mathrm{F} \beta$ con alta turbulencia

La figura 11a muestra el observable eléctrico $V_{O}$ recibido en turbulencia débil, y la salida de cada uno de los bloques "estimador-correlacionador" tanto de " 0 " (fi-

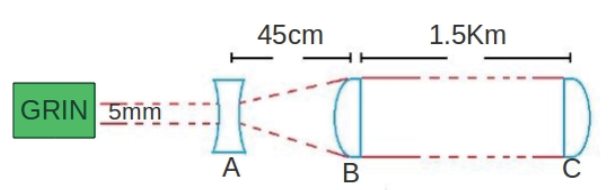

Lente A: diámetro 6, long. focal 50, radio curv. 9.74 [mm] Lente B: diámetro 50, long. focal 500, radio curv. 258.4 [mm] Lente C: diámetro 25 , long. focal 25 , radio curv. $16.82[\mathrm{~mm}]$

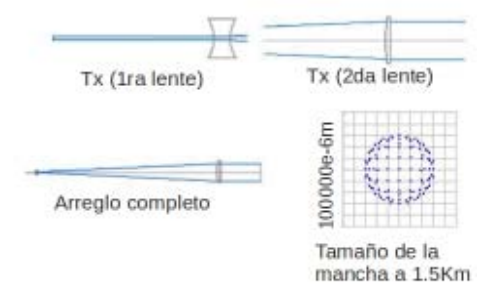

Figura 8. a) Arreglo de antenas ópticas, b) simulación en ZEMAX 

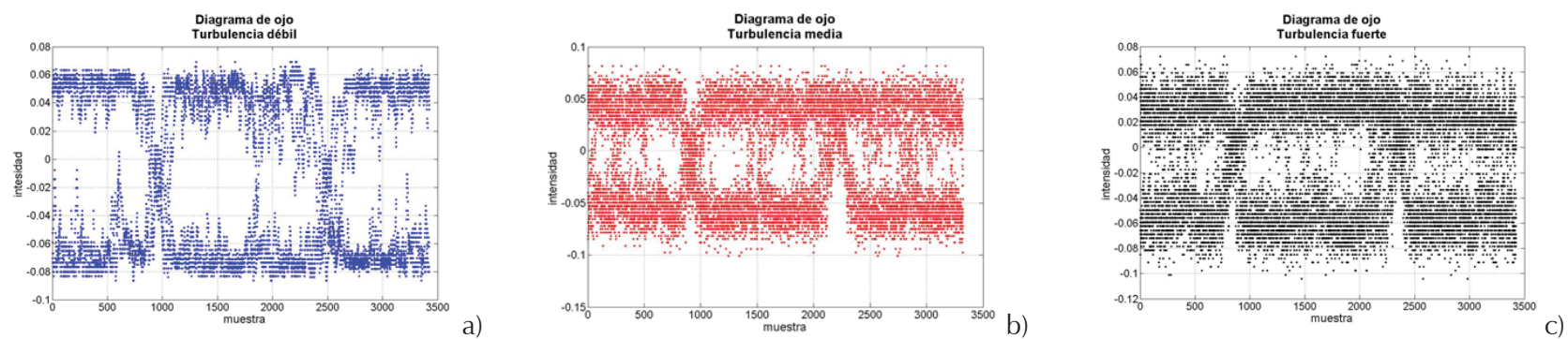

Figura 9. Diagramas de ojo obtenidos para turbulencia, a) débil, b) media, c) fuerte

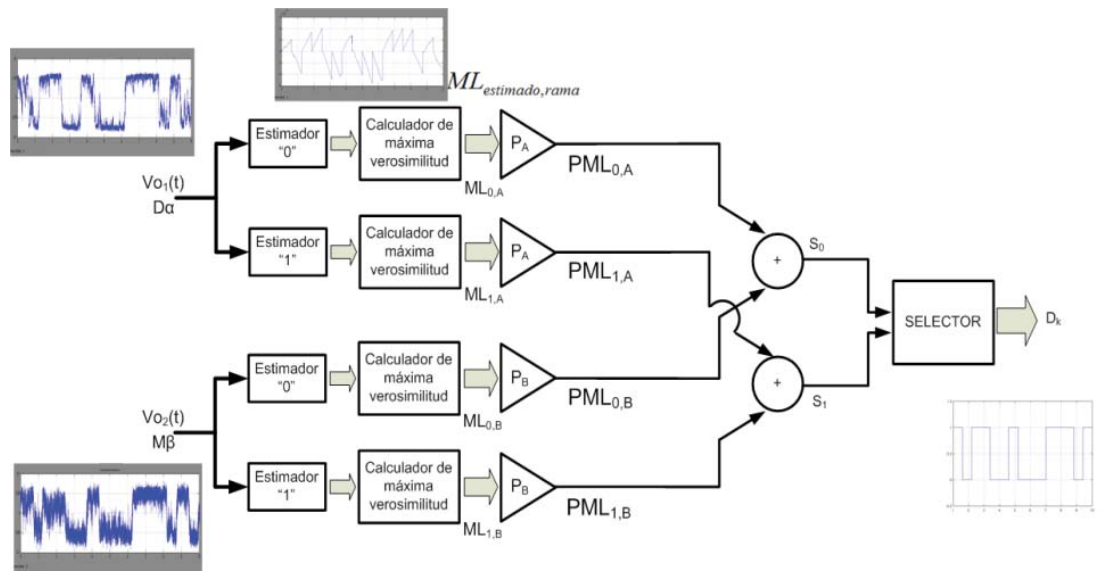

Figura 10. Estimador-correlacionador para un sistema de diversidad espacial grado 2
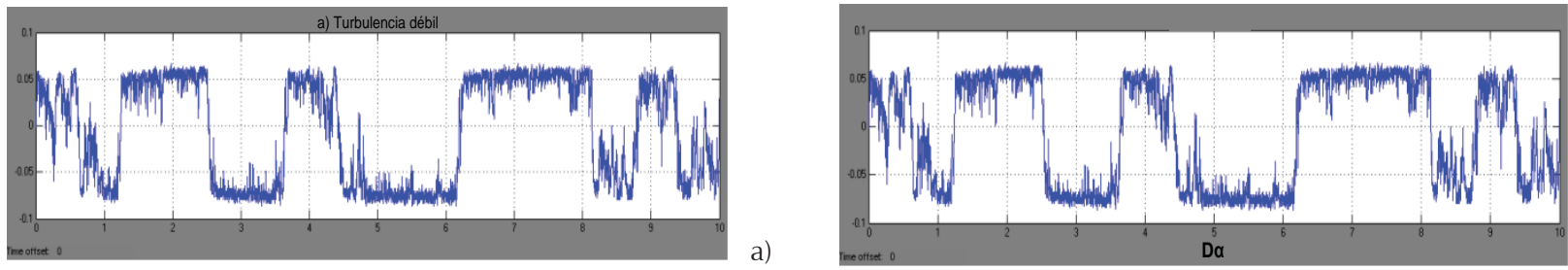

d)

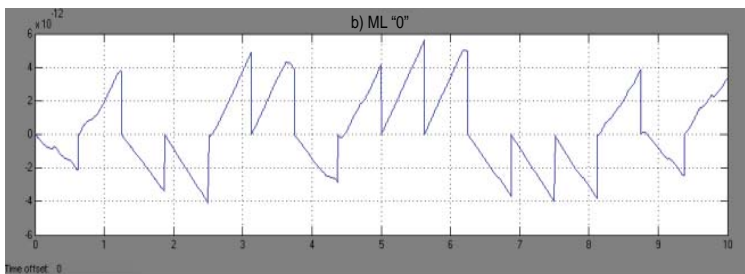

a)

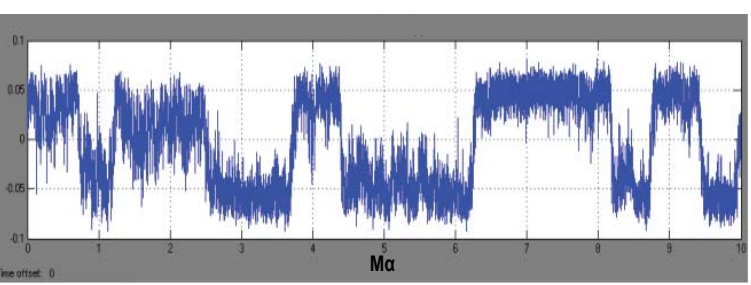

e)
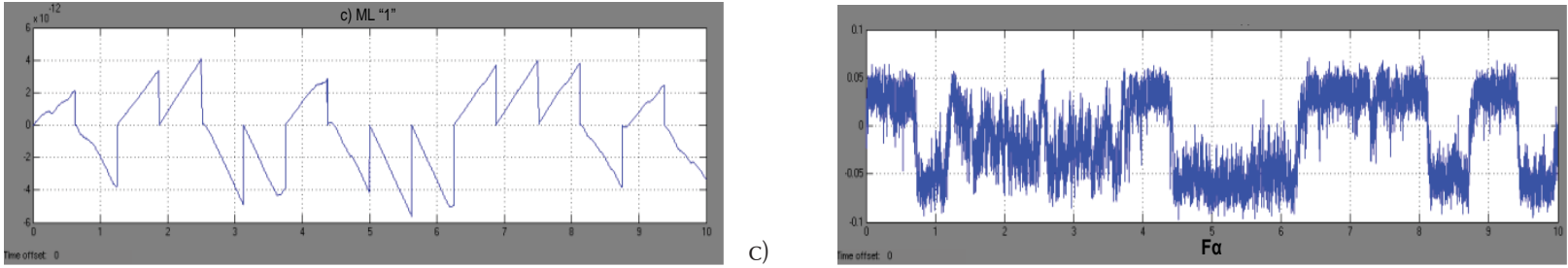

f)

Figura 11. a) Observable eléctrico (turbulencia débil), b) salida del estimador-correlacionador para “ 0 ", c) salida del estimadorcorrelacionador para " 1 ", d)-f) tres observables eléctricos $\left(V_{\mathrm{O}}\right)$ adquiridos para su procesamiento digital 
gura 11b) como de "1" (figura 11c), indicados en la figura como las señales $M_{0} \mathrm{y} \mathrm{ML}_{1}$.

La manera en la que se agruparon estas señales para la conformación de diversos escenarios con diversidad espacial se muestra en la tabla 2.

De esta manera se plantean tres casos diferentes para un esquema con diversidad espacial $2 \times 2$ (figura 10), la razón de agrupar así los casos se basa en que cada una de las ramas de un sistema de comunicaciones con diversidad espacial representa un canal independiente $\mathrm{y}$, por tanto, es factible el hecho de que cada rama sufra un nivel de turbulencia distinto. Como se mencionó anteriormente, es necesario determinar los pesos de cada una de las ramas, para el esquema $2 \times 2$ se calcularán tantas intensidades totales como casos y en cada caso existen tantos pesos a determinar como ramas. Para la determinación de los pesos la primera operación realizada fue el cálculo de la intensidad promedio de cada trama de datos.
De esta manera se importan las señales $\mathrm{D} \alpha$ y $\mathrm{M} \beta$ al programa elaborado en SIMULINK, el cual entrega los valores de los estimados " 0 " $\mathrm{y}$ " 1 " $\left(\mathrm{ML}_{0, \mathrm{~A}^{\prime}}, \mathrm{ML}_{1, \mathrm{~A}^{\prime}} \mathrm{ML}_{0, \mathrm{~B}}\right.$ $\mathrm{ML}_{1, \mathrm{~B}}$ ) para cada señal, estos valores deben ahora ser ponderados por los pesos anteriormente calculados para así obtener las señales $\left(\mathrm{PML}_{0, \mathrm{~A}} \mathrm{PML}_{1, \mathrm{~A}^{\prime}} \mathrm{PML}_{0, \mathrm{~B}}\right.$ $\mathrm{PML}_{1, \mathrm{~B}}$ ), posteriormente se realizó la suma correspondiente para la obtención de los valores de $\mathrm{S}_{0} \mathrm{y}_{1}$, ambas variables se comparan bit a bit y el mayor de estos es el dato estimado $D_{k}$.

En la tabla 3 se muestran los resultados obtenidos para el caso 1 de la tabla 2 y una trama transmitida de bits = (1 0110010001111010$)$. Un punto importante es que mediante esta estrategia es posible implementar otros esquemas, de hecho lo llevamos a cabo para un esquema $4 \times 4$ y en la tabla 4 se resumen los resultados obtenidos para ambos casos $(2 \times 2$ y $4 \times 4)$.

Tabla 2. Casos para analizar en un esquema de diversidad espacial $2 \times 2(\mathrm{~A} 2, \mathrm{~B} 2)$ y $4 \times 4(\mathrm{~A} 4, \mathrm{~B} 4, \mathrm{C} 4, \mathrm{D} 4)$

\begin{tabular}{lcccccc}
\hline & Rama A2 & Rama B2 & Rama A4 & Rama B4 & Rama C4 & Rama D4 \\
\hline Caso \#1 & $\mathrm{D} \alpha$ & $\mathrm{M} \beta$ & $\mathrm{D} \alpha$ & $\mathrm{D} \beta$ & $\mathrm{M} \alpha$ & $\mathrm{F} \alpha$ \\
Caso \#2 & $\mathrm{D} \beta$ & $\mathrm{F} \alpha$ & $\mathrm{D} \beta$ & $\mathrm{M} \alpha$ & $\mathrm{M} \beta$ & $\mathrm{F} \beta$ \\
Caso \#3 & $\mathrm{M} \alpha$ & $\mathrm{F} \beta$ & $\mathrm{M} \beta$ & $\mathrm{F} \alpha$ & $\mathrm{F} \beta$ & $\mathrm{D} \alpha$ \\
Caso \#4 & & & $\mathrm{M} \alpha$ & $\mathrm{M} \beta$ & $\mathrm{F} \alpha$ & $\mathrm{F} \beta$ \\
\hline
\end{tabular}

Tabla 3. Dato estimado para el caso \#1 bajo un esquema transmisión $2 \times 2$

\begin{tabular}{|c|c|c|c|c|c|c|c|c|c|c|}
\hline \multicolumn{8}{|c|}{ CASO \#1, ESQUEMA 2 X2 } & \multirow{4}{*}{$\begin{array}{c}\mathrm{S}_{0}= \\
\mathrm{PML}_{0, \mathrm{~A}} \\
+\mathrm{PML}_{0, \mathrm{~B}}\end{array}$} & \multirow{4}{*}{$\begin{array}{c}\mathrm{S}_{1}= \\
\mathrm{PML}_{1, \mathrm{~A}} \\
+ \\
\mathrm{PML}_{1, \mathrm{~B}}\end{array}$} & \multirow[b]{4}{*}{$D_{k}$} \\
\hline \multirow{2}{*}{\multicolumn{4}{|c|}{$\begin{array}{c}\text { Rama A } \\
\mathbf{P}_{1, \mathrm{~A}}=0.49868957\end{array}$}} & \multirow{2}{*}{\multicolumn{4}{|c|}{$\begin{array}{c}\text { Rama B } \\
\mathbf{P}_{1, \mathrm{~B}}=0.50131042\end{array}$}} & & & \\
\hline & & & & & & & & & & \\
\hline $\mathrm{ML}_{0, \mathrm{~A}}$ & $\mathrm{ML}_{1, \mathrm{~A}}$ & $\mathrm{PML}_{0, \mathrm{~A}}$ & $\mathrm{PML}_{1, \mathrm{~A}}$ & $\mathbf{M L}_{0, \mathrm{~B}}$ & $\mathrm{ML}_{1, \mathrm{~B}}$ & $\mathrm{PML}_{0, \mathrm{~B}}$ & $\mathrm{PML}_{1, \mathrm{~B}}$ & & & \\
\hline 2.09 & 2.12 & 1.042 & 1.057 & 2.06 & 2.11 & 1.032 & 1.057 & 2.074 & 2.114 & 1 \\
\hline 3.7 & 3.65 & 1.845 & 1.820 & 2.64 & 2.59 & 1.323 & 1.298 & 3.168 & 3.118 & 0 \\
\hline 3.99 & 4.01 & 1.989 & 1.999 & 3.84 & 3.9 & 1.925 & 1.955 & 3.914 & 3.954 & 1 \\
\hline 4.73 & 4.77 & 2.358 & 2.378 & 1.79 & 1.84 & 0.897 & 0.922 & 3.256 & 3.301 & 0 \\
\hline 4.21 & 4.18 & 2.099 & 2.084 & 1.72 & 1.68 & 0.862 & 0.842 & 2.961 & 2.926 & 0 \\
\hline 3.91 & 3.88 & 1.949 & 1.934 & 1.65 & 1.61 & 0.827 & 0.807 & 2.777 & 2.742 & 0 \\
\hline 2.61 & 2.76 & 1.301 & 1.376 & 2.13 & 2.19 & 1.067 & 1.097 & 2.369 & 2.474 & 1 \\
\hline 4.03 & 4 & 2.009 & 1.994 & 3.88 & 3.81 & 1.945 & 1.909 & 3.954 & 3.904 & 0 \\
\hline 5.7 & 5.63 & 2.842 & 2.807 & 4.58 & 4.54 & 2.296 & 2.275 & 5.138 & 5.083 & 0 \\
\hline 4.85 & 4.84 & 2.418 & 2.413 & 3.75 & 3.69 & 1.879 & 1.849 & 4.298 & 4.263 & 0 \\
\hline 3.66 & 3.71 & 1.825 & 1.850 & 2.1 & 2.18 & 1.052 & 1.092 & 2.877 & 2.942 & 1 \\
\hline 3.88 & 3.93 & 1.934 & 1.959 & 2.55 & 2.61 & 1.278 & 1.308 & 3.213 & 3.268 & 1 \\
\hline 3.79 & 3.82 & 1.890 & 1.904 & 2.3 & 2.35 & 1.153 & 1.178 & 3.043 & 3.083 & 1 \\
\hline 3.79 & 3.76 & 1.890 & 1.875 & 3.85 & 3.8 & 1.930 & 1.904 & 3.820 & 3.780 & 0 \\
\hline 2.67 & 2.71 & 1.331 & 1.351 & 2.48 & 2.52 & 1.243 & 1.263 & 2.574 & 2.614 & 1 \\
\hline 3.31 & 3.28 & 1.650 & 1.635 & 3.68 & 3.61 & 1.844 & 1.809 & 3.495 & 3.445 & 0 \\
\hline
\end{tabular}


Tabla 4. Comparación entre la secuencia enviada y la secuencia estimada en todos los casos planteados

\begin{tabular}{cccccccccccccccccc}
\hline & dato & 1 & 0 & 1 & 1 & 0 & 0 & 1 & 0 & 0 & $\mathbf{0}$ & $\mathbf{1}$ & $\mathbf{1}$ & $\mathbf{1}$ & $\mathbf{0}$ & $\mathbf{1}$ & $\mathbf{0}$ \\
\hline $\begin{array}{c}\text { casos } \\
\text { para }\end{array}$ & $\# \mathbf{1}$ & $\checkmark$ & $\checkmark$ & $\checkmark$ & $\mathbf{x}$ & $\checkmark$ & $\checkmark$ & $\checkmark$ & $\checkmark$ & $\checkmark$ & $\checkmark$ & $\checkmark$ & $\checkmark$ & $\checkmark$ & $\checkmark$ & $\checkmark$ & $\checkmark$ \\
esquema & $\# \mathbf{2}$ & $\checkmark$ & $\checkmark$ & $\checkmark$ & $\checkmark$ & $\checkmark$ & $\checkmark$ & $\checkmark$ & $\checkmark$ & $\checkmark$ & $\checkmark$ & $\checkmark$ & $\checkmark$ & $\checkmark$ & $\checkmark$ & $\checkmark$ & $\checkmark$ \\
$\mathbf{2 x 2}$ & $\# \mathbf{3}$ & $\checkmark$ & $\checkmark$ & $\checkmark$ & $\checkmark$ & $\checkmark$ & $\checkmark$ & $\checkmark$ & $\checkmark$ & $\checkmark$ & $\checkmark$ & $\checkmark$ & $\checkmark$ & $\checkmark$ & $\checkmark$ & $\checkmark$ & $\checkmark$ \\
& $\# \mathbf{1}$ & $\checkmark$ & $\checkmark$ & $\checkmark$ & $\checkmark$ & $\checkmark$ & $\checkmark$ & $\checkmark$ & $\checkmark$ & $\checkmark$ & $\checkmark$ & $\checkmark$ & $\checkmark$ & $\checkmark$ & $\checkmark$ & $\checkmark$ & $\checkmark$ \\
$\begin{array}{c}\text { casos } \\
\text { para }\end{array}$ & $\# \mathbf{2}$ & $\checkmark$ & $\checkmark$ & $\checkmark$ & $\checkmark$ & $\checkmark$ & $\checkmark$ & $\checkmark$ & $\checkmark$ & $\checkmark$ & $\checkmark$ & $\checkmark$ & $\checkmark$ & $\checkmark$ & $\checkmark$ & $\checkmark$ & $\checkmark$ \\
$\begin{array}{c}\text { esquema } \\
\text { 4x4 }\end{array}$ & $\# 3$ & $\checkmark$ & $\checkmark$ & $\checkmark$ & $\checkmark$ & $\checkmark$ & $\checkmark$ & $\checkmark$ & $\checkmark$ & $\checkmark$ & $\checkmark$ & $\checkmark$ & $\checkmark$ & $\checkmark$ & $\checkmark$ & $\checkmark$ & $\checkmark$ \\
& $\# \mathbf{4}$ & $\checkmark$ & $\checkmark$ & $\checkmark$ & $\checkmark$ & $\checkmark$ & $\checkmark$ & $\checkmark$ & $\checkmark$ & $\checkmark$ & $\checkmark$ & $\checkmark$ & $\checkmark$ & $\checkmark$ & $\checkmark$ & $\checkmark$ & $\checkmark$ \\
\hline
\end{tabular}

\section{Conclusiones}

En este trabajo reportamos la caracterización experimental de un sistema de comunicaciones ópticas MIMO homodino tolerante a difusión de fase óptica y turbulencia atmosférica. El sistema reportado es una versión simplificada de laboratorio que permite evaluar el desempeño del algoritmo de diversidad espacial y estimación óptima de fase óptica postdetección. Por razones de espacio, no se describe el criterio de diseño que optimiza la tolerancia al ruido de fase, para lo cual, nos basamos en el trabajo de Arvizu (2010). Como parte del montaje experimental se implementó una cámara para la generación controlada de turbulencia óptica, se diseñaron las antenas ópticas, auxiliados con el software ZEMAX. La existencia de las perturbaciones descritas en este trabajo (ruido de fase y turbulencia óptica) dificulta enormemente la implementación de sistemas como el reportado. Así, el poder pasar de modelos de simulación a implementaciones experimentales a nivel laboratorio (y eventualmente a prototipos) es un paso muy importante, de allí la conveniencia de tener resultados no solamente teóricos o de simulación, sino poder mostrar la factibilidad de realización práctica de dichos sistemas. Podemos concluir que bajo las condiciones de operación reportadas, el sistema funciona satisfactoriamente y con el propósito de que el sistema completo pueda trabajar en tiempo real se requieren tanto de etapas de conversión A/D como de procesadores digitales de señales o FPGA's de alta velocidad

\section{Agradecimientos}

Este trabajo fue desarrollado con fondos de un proyecto de Ciencia Básica del CONACYT.

\section{Referencias}

Andrew L.C. y Phillips R.L. Laser beam propagation through random media, Bellingham, Washington, SPIE Press, 2005, pp.782.

Arvizu-Mondragón A., Sánchez-López J.D.D., Mendieta-Jiménez F.J. Coherent optical wireless link employing phase estimation with multiple-beam, multiple-aperture, for increased tolerance to turbulence. IEICE Transactions on Communications, volumen E93-B (número 1), 2010: 226-229.

Bell A.G. The production of sound by radiant energy. Science, volumen 2 (número 48), mayo 28 de 1881: 242-253.

Belmonte A. y Kahn J.M. Capacity of coherent free-space optical links using diversity-combining techniques. Optics Express, volumen 17 (número 15), 2009: 12601-12611[en línea]. Disponible en: https://www.osapublishing.org/oe/fulltext.cfm?uri= oe-17-15-12601\&id=183574

Farid A.A. y Hranilovic S. Diversity gain and outage probability for MIMO free-space optical links with misalignment. IEEE Transactions on Communications, volumen 60 (número 2), febrero de 2012: 479-487.

Goyal P., Kumar A., Nath V. Mitigation of atmospheric turbulence in free space optics: a review, en:0 IEEE 2015 Fifth International Conference on Advanced Computing \& Communication Technologies (ACCT), 21-22 de febrero de 2015, Haryana, India, pp. 634-640.

Grosinger J. Investigation of polarization modulation in optical free space communications through the atmosphere, tesis (ingeniería), Instituto de Comunicación y de Ingeniería de Radio-Frecuencia, Viena, Austria, 2008. 83 p.

Kashani M.A., Safari M., Uysal M. Optimal relay placement and diversity analysis of relay-assisted free-space optical communication systems. Journal of Optical Communications and Networking, volumen 5 (número 1), 2013: 37-47.

Khalighi M.A. y Uysal M. Survey on free space optical communication: a communication theory perspective. IEEE Communi- 
cation Surveys \& Tutorials, volumen 16 (número 4), 2014: 2231-2258.

Hajjarian Z. y Fadlullah J. MIMO free space optical communications in turbil and turbulent atmosphere. Journal of communications, volumen 4 (número 8), 2009: 524-532.

Lighpointe homepage [en línea]. Disponible en: http://www.lightpointe.com (fecha de consulta: 17 de julio 2015).

Niu M., Schlenker J., Cheng J., Holzman J.F., Schober R., Coherent wireless optical communications with predetection and postdetection EGC over gamma-gamma atmospheric turbulence channels. IEEE/OSA Journal of Optical Communications and Networking, volumen 3 (número 11), 2011: 860-869.

Popoola W.O., Ghassemlooy Z., Allen J.I.H., Leitgeb E., Gao S. Free space optical communication employing modulation and spatial diversity in atmospheric turbulence channel. IET Optoelectron, volume 2 (número 1), 2008: 16-23.

Prabu K., Kumar D.S. MIMO free-space optical communication employing coherent BPOLSK modulation in atmospheric optical turbulence channel with pointing errors. Optics Communications, volumen 343, 2015: 188-194.
Snyder D.L. The state variable approach to continuous estimation, Res. Mon., Núm. 51, Cambridge, Massachusetts, MIT Press, 1969, p.114.

\section{Este artículo se cita:}

\section{Citación estilo Chicago}

Arvizu-Mondragón, Arturo, Joel Santos-Aguilar, Alejandro Cabrera-Nava. Sistema MIMO óptico inalámbrico con modulación BPSK y recepción óptima homodina tolerante a difusión de fase óptica y turbulencia atmosférica. Ingeniería Investigación y Tecnología, XVII, 02 (2016): 283-295.

\section{Citación estilo ISO 690}

Arvizu-Mondragón A., Santos-Aguilar J., Cabrera-Nava A. Sistema MIMO óptico inalámbrico con modulación BPSK y recepción óptima homodina tolerante a difusión de fase óptica y turbulencia atmosférica. Ingeniería Investigación y Tecnología, volumen XVII (número 2), abril-junio 2016: 283-295.

\section{Semblanzas de los autores}

Arturo Arvizu-Mondragón. Recibió el grado de ingeniero mecánico electricista por la Facultad de Ingeniería, UNAM, en 1987, obtuvo la maestría en ingeniería eléctrica con especialidad en comunicaciones en la DEPFI-UNAM, en 1990. Doctorado en ciencias en electrónica y telecomunicaciones, CICESE, Ensenada, BC, México en 2000. En 2001 realizó una estancia posdoctoral en el Departamento de Telecomunicaciones de la École Nationale Supérieure des Télécommunications, Paris, Francia. Desde 1987 a febrero 1992 trabajó en el Instituto de Investigaciones Eléctricas en Cuernavaca, México en proyectos de sistemas de comunicaciones optoelectrónicas y pruebas de medición y caracterización de sistemas eléctricos. Desde marzo1992 hasta la fecha realiza trabajo de investigación en CICESE, sus principales líneas de investigación son: comunicaciones ópticas inalámbricas, comunicaciones cuánticas, comunicaciones por fibra óptica con detección coherente.

Joel Santos Aguilar. Recibió el grado de licenciatura en electrónica por la Benemérita Universidad Autónoma de Puebla, México, en 2002. Obtuvo el grado de maestría en ciencias, en electrónica en 2005 por el Instituto Nacional de Astrofísica Óptica y Electrónica (INAOE), México, y el doctorado en ciencias en óptica en 2009 por INAOE, México. Realizó de julio 2012 a junio 2013 una estancia posdoctoral de investigación en CICESE, Baja California México, donde actualmente es investigador y sus líneas de trabajo son principalmente las comunicaciones ópticas por fibra óptica y espacio libre así como radio sobre fibra.

Alejandro Cabrera-Nava. Egresado de la Facultad de Ciencias de la Electrónica de la Universidad Autónoma de Puebla (BUAP) en el año 2003, obtuvo el grado de maestría en 2012 en el Centro de Investigación Científica y Educación Superior de Ensenada (CICESE) en el área de telecomunicaciones ópticas. Trabajó en Ericsson en la implementación de proyectos de RF en Sudamérica y actualmente labora en la división de comunicaciones ópticas de Huawei para la región Norteamericana. 